\title{
La reproduction sexuée et l'agressivité de Phaeo- sphaeria nodorum Hedj (= Septoria nodorum Berk)
}

\author{
F Rapilly ${ }^{1}$, M Skajennikoff 1 , P Halama 2, G Touraud 1 \\ 1 INRA, station de pathologie végétale, route de St-Cyr, 78026 Versailles; \\ 2 ISA, 41 rue du Port, 59046 Lille, France
}

(Reçu le 3 avril 1992; accepté le 7 juillet 1992)

\begin{abstract}
Résumé - La variabilité de l'agressivité, de Septoria nodorum, parasite du blé, a été l'objet de nombreuses études. Cet article présente la variabilité obtenue, chez ce parasite, après reproduction sexuée. Les 8 souches monoascospores provenant d'un même asque de Phaeosphaeria nodorum sont comparées, sur diverses variétés de blé, par l'appréciation sur feuilles détachées de la progression des nécroses foliaires et par la mesure de la quantité d'éthylène produite après contamination. Ce champignon est hétérothallique; la comparaison des 2 groupes d'incompatibilité sexuelle montre que ceux-ci sont en moyenne d'agressivité comparable; au sein de chaque groupe, les amplitudes obtenues entre les ascospores sont de même grandeur. Les 4 paires d'ascospores jumelles reconnues sur la base des caractères culturaux ne sont pas identifiables sur la base des critères utilisés pour les feuilles en survie ou sur la base de la progression des épidémies. Par le pouvoir pathogène, la reconnaissance des ascospores jumelles se révèle impossible. La descendance conidienne d'une même ascospore est hétérogène. Des hypothèses sont formulées pour expliquer, d'une part, la non-reconnaissance des jumeaux, et d'autre part, l'hétérogénéité de la descendance d'une ascospore.
\end{abstract}

agressivité / ascospore / blé / variabilité / Phaeosphaeria nodorum / Septoria nodorum

Summary - Sexual reproduction and aggressiveness of Phaeosphaeria nodorum Hedj (= Septoria nodorum Berk). The variability in aggressiveness of Septoria nodorum, a wheat pathogen, has been extensively studied. This article presents the variability obtained in this pathogen after sexual reproduction. The 8 mono-ascosporous strains originating from the same Phaeosphaeria nodorum ascus were compared by assessing the progression of leaf necroses on detached leaves of various wheat varieties and by measuring the quantity of ethylene produced after contamination. This fungus is heterothallic; the comparison between the 2 sexually incompatible groups showed that on average they are similarly aggressive. In each group the ranges obtained between ascospores were of the same size. The 4 pairs of twin ascospores identified on the basis of cultural characteristics were not identifiable on the basis of criteria used for detached leaves, or of the progression of epidemics. For pathogenicity, identification of twin ascospores was impossible. Conidian progeny of the same ascospore was shown to be heterogeneous. Hypotheses have been proposed to explain the non-identification of twins and, also, the heterogeneity of the ascospore progeny.

aggressiveness / ascospores / wheat / instability / Septoria nodorum / Phaeosphaeria nodorum

\section{INTRODUCTION}

De nombreux travaux rassemblés par Nelson et Marshall (1990), montrent que l'agressivité d'isolements de blé de Septoria nodorum Berk (= Phaeosphaeria nodorum Hedj) provenant de diverses origines, est éminemment variable. II en est de même entre pycnides provenant d'un même isolat, mais aussi entre pycnidiospores provenant d'une même pycnide (Krupinsky, 1982; Osbourn et al, 1986). Cette hétérogénéité d'une pycnide est si grande que son contenu est assimilable, pour l'agressivité, à une population (Skajennikoff et Rapilly, 1989). La reproduction conidienne permet, après une seule généra- tion, la détection des pressions de sélection exercées par un hôte sur ce parasite (Rapilly et al, 1989).

L'amplitude de l'agressivité après la reproduction sexuée de ce parasite n'a pas été étudiée en détail. Rapilly et al (1973) constatent des différences significatives du pouvoir pathogène entre ascospores de Phaeosphaeria nodorum récoltées sur chaumes de blé. Halama (1991) montre, après avoir étudié plusieurs séries d'ascospores obtenues in vitro, que la reproduction sexuée permet d'obtenir une plage d'agressivité qui s'étend au-delà et en deçà des niveaux que représentent les souches parentales. Cet auteur note que les différents critères utilisés pour apprécier l'agressi- 
vité ne permettent pas d'identifier les ascospores qui sont des jumelles nucléaires.

Chez les Ascomycètes la différenciation des 8 noyaux qui donneront ultérieurement les 8 ascospores est obtenue, après formation du zygote, par 3 divisions nucléaires successives. Les 2 premières sont une division méiotique réductionnelle suivie d'une division méiotique équationnelle; la troisième est une mitose haploïde (Jinks, 1966). Phaeosphaeria nodorum est un organisme hétérothallique (Halama et Lacoste, 1990). Le produit de la méiose peut être identifié par la connaissance de l'allèle d'incompatibilité sexuelle de chaque ascospore : 2 groupes d'incompatibilité formés de 4 ascospores chacun peuvent être considérés. Le produit de la dernière mitose peut être reconnu par l'identification des quatre paires d'ascospores nucléairement jumelles.

Les résultats présentés correspondent à l'étude de l'agressivité des 8 ascospores issues du même asque. Ces ascospores sont regroupées par groupe d'incompatibilité et par paire de jumelles.

\section{MATÉRIEL ET MÉTHODES}

\section{Matériel fongique}

Les 8 ascospores dénommées $\times 1, \times 2 \ldots \times 8$, proviennent du même asque qui est issu d'un croisement réalisé par Halama (1991) entre une ascospore A $/ 5$, isolée d'un périthèce trouvé sur chaume de blé dans le nord de la France, et la souche $6 / \mathrm{T}$ dérivée de la souche 6 par un passage sur le triticale T 762 (Rapilly et al, 1989). La souche 6 a été isolée du blé Étoile de Choisy à Versailles. Ces deux souches parentales apparaissent très différentes l'une de l'autre (tableau I). Elles sont de signe sexuel complémentaire et diffèrent par leur origine, la pigmentation mycélienne des cultures, leur agressivité sur feuilles de blé, la réduction du poids de 1000 grains qu'elles provoquent. La croissance mycélienne de $6 / \mathrm{T}$ in vitro est supérieure à celle de $A / 5$, ainsi que son aptitude à la sporulation.

Les ascospores sont isolées par micromanipulation (micromanipulateur de Fontbrune) et de ce fait leur ordre dans l'asque n'est pas connu. Cette ignorance de la disposition dans l'asque des allèles de signes sexuels ne permet pas de savoir laquelle des 2 premières divisions nucléaires est réductionnelle (Emerson, 1966). L'appartenance des ascospores à tel ou tel groupe d'incompatibilité (tableau II) est déterminée par la réussite des croisements entre les ascospores confrontées 2 à 2 et avec d'autres ascospores au signe sexuel connu.

L'identification des 4 paires d'ascospores jumelles nucléaires est faite, sans aucune ambiguité, sur la base des caractères culturaux pour des cultures réalisées, in vitro, dans les mêmes conditions. La pigmentation mycélienne permet facilement cette reconnaissance (tableau II). Dans d'autres séries d'ascospores, Halama (1991) montre que seul ce caractère permet d'associer les ascospores par paires de jumelles. Cette modalité d'identification présuppose que l'aspect cultural est sous la dépendance d'une hérédité nucléaire. Keitt et Langford (1941) utilisent ce même critère pour reconnaître les ascospores jumelles de Venturia inaequalis, qui est aussi un organisme à hétérothallisme bipolaire.

A partir d'une pycnide issue de l'ascospore $x 6$ et d'une autre issue de l'ascospore $\times 8,5$ isolements monomacropycnidiospores sont réalisés. Les souches

Tableau I. Caractéristiques des souches parentales du croisement étudié.

Caractéristiques relevées

\begin{tabular}{cc}
\multicolumn{2}{c}{ Parents } \\
\hline$A / 5$ & $6 / \tau$
\end{tabular}

Signe sexuel

Pigmentation des cultures in vitro

Longueur (en mm) des nécroses foliaires après $10 \mathrm{j}$ à $18^{\circ} \mathrm{C} 1$

Sur Camp Rémy

Sur Étoile de Choisy

Sur la lignée $L_{22}$

Réduction en (\%) du poids de 1000 grains après contamination à l'anthèse

Sur Étoile de Choisy ( $1^{\text {er }}$ essai)

Sur Étoile de Choisy ( $2^{\mathrm{e}}$ essai)

Sur la lignée UPC $_{5}$ (1 $1^{\text {er essai) }}$

Sur la lignée $\mathrm{UPC}_{5}\left(2^{\mathrm{e}}\right.$ essai)
$(+)$

Gris verdâtre

$\begin{array}{lr}4,63 & 6,73 \\ 6,72 & 10,65 \\ 3,31 & 4,53 \\ & \\ 30,3 & 31,97 \\ 34,61 & 39,31 \\ 29,36 & 24,23 \\ 23,94 & 20,35\end{array}$

$(-)$

Blanc rosé

6,73

(6,65

31,97

24,23

20,35

\footnotetext{
1 Les valeurs relevées diffèrent entre elles, au seuil de 0,05 au minimum.
} 
Tableau II. Groupes de compatibilité sexuelle (+ ou -) et pigmentation mycélienne des paires d'ascospores jumelles issues d'une même asque.
Groupe +
Groupe -

$x 1-x 8=$ vert jaunâtre

$x 2-x 3=$ jaune verdâtre

$x 5-x 7=$ gris verdâtre

$x 4-x 6=$ blanc rosé

obtenues sont dénommées $\times 6 . P_{3}$.. pour l'ascospore $\times 6$ et $\times 8 . P_{1}$.. pour l'ascospore $x 8$, suivi du numéro de la monospore. Toutes les souches issues de la même ascospore ont un aspect cultural identique entre elles.

La sporulation des cultures est obtenue en boîte de Petri plastique, sur le milieu M 23 modifié (Halama, 1991). Ces cultures sont conduites à $14{ }^{\circ} \mathrm{C}$ sous une photopériode de $12 \mathrm{~h} / 24 \mathrm{~h}$. Un éclairement de 5000 lux, au niveau des cultures, est obtenu par la disposition alternée de tubes fluorescents Sylvania blanc industrie et Grolux; ces derniers apportent des rayonnements proches UV. Des suspensions titrant $10^{6}$ spores par $\mathrm{ml}$ sont utilisées pour réaliser toutes les inoculations faites in vitro ou in vivo.

\section{Matériel végétal}

L'agressivité des souches est jugée sur fragments foliaires de $4 \mathrm{~cm}$ de long, prélevés dans la partie centrale des $3^{e}$ et $4^{e}$ feuilles de plantules de blé élevées en serre. Les variétés ou lignées utilisées sont : Étoile de Choisy et $\mathrm{UPC}_{5}$ très sensibles, $\mathrm{L}_{22}$ et $\mathrm{CBS}_{1}$ à résistance foliaire élevée et Camp Rémy de résistance intermédiaire.

Au champ, les épidémies sont suivies sur des parcelles de $2 \mathrm{~m}^{2}$ de la variété Étoile de Choisy et de la lignée $\mathrm{UPC}_{5}$.

\section{Expérimentations et notations}

\section{Sur fragments foliaires en survie}

Les comparaisons entre les souches monoascosporées et les souches monomacropycnidiospores sont réalisées dans des essais blocs comprenant au minimum quatre répétitions. Les fragments foliaires sont maintenus en survie sur une solution de benzimidazole à $50 \mathrm{ppm}$; ils sont disposés, à une température moyenne de $17^{\circ} \mathrm{C}$, sous un éclairement de $12 / 24 \mathrm{~h}$. Les inoculations sont obtenues par dépôt, sur la face supérieure et dans la partie centrale de chaque fragment, de deux microgouttes calibrées à $20 \mu$ d'une suspension de spores. Ces conditions, reproductibles d'un essai à l'autre, permettent de s'affranchir des effets du milieu. Pour un essai, la longueur des nécroses foliaires est mesurée après $10 \mathrm{j}$; pour les autres, cette longueur et l'intensité des nécroses sont appréciées avec une échelle de notation de 0 à 5 ( 0 correspond à une absence visuelle de nécrose, 5 à une nécrose au centre très marquée et qui atteint, après $5 \mathrm{j}, 15 \mathrm{~mm}$ de long).

Dans un essai conduit sur fragments d'Étoile de Choisy, la quantité d'éthylène produite à $24,48,72$ et $96 \mathrm{~h}$ après inoculation, par dépôt de 4 micro-gouttes de $5 \mu \mathrm{l}$ d'une suspension de spores, a été mesurée en suivant la méthodologie proposée par Wendland et Hoffmann $(1987,1988)$. La quantité d'éthylène produite est exprimée en $\mathrm{nl}$ pour $100 \mathrm{mg}$ de poids frais de fragments foliaires. L'intensité et la taille des nécroses foliaires sont relevées aux mêmes périodes de temps.

\section{Au champ}

La progression des épidémies est suivie, pendant 58 j, dans un essai bloc à 2 répétitions par le relevé, à des dates régulières (7 notations), du pourcentage de surface foliaire malade après la contamination réalisée à la mi-montaison. Le critère de l'aire sous la courbe de progression de l'épidémie, en fonction du temps, est celui retenu pour comparer les souches monoascospores.

\section{Interprétations}

L'homogénéité entre les répétitions de chaque essai et les différences entre les souches sont jugées au travers d'analyses de variance. Si la valeur du $F$ est significative, la ppds est calculée pour distinguer les souches.

Les groupes d'incompatibilité sexuelle qui regroupent chacun 4 ascospores sont comparés en jugeant, par un test de $t$, de la signification de la différence à partir des valeurs moyennes obtenues pour chaque ascospore; dans ce cas, il y a 6 ddl; les groupes sont d'effectifs égaux mais de variances inégales. Les ascospores jumelles sont comparées, pour chaque paire, suivant le même principe, mais à partir des données obtenues pour chaque répétition d'un essai; dans ce cas, le nombre de degrés de liberté est égal au nombre d'observations moins 2 . L'ensemble des paires jumelles est comparé par un test de $t$, pour juger de la signification de la différence entre les valeurs absolues moyennes de chaque paire d'ascospores, le nombre de ddl est de 3 , car les observations sont considérées comme appariées.

\section{RÉSULTATS}

\section{Nécroses sur feuilles en survie}

Pour 3 variétés de blé, la longueur nécrosée relevée en $\mathrm{mm}$ après 10 j montre des différences hautement significatives entre les ascospores et pour chaque variété (tableau IIla). L'amplitude 
Tableau Illa. Longueur (en $\mathrm{mm}$ ) des nécroses foliaires sur fragments foliaires maintenus en survie après $10 \mathrm{j}$ à $17^{\circ} \mathrm{C}$ (moyenne de 8 répétitions).

\begin{tabular}{|c|c|c|c|c|c|c|c|c|c|c|c|}
\hline \multirow[t]{2}{*}{ Variétés } & \multicolumn{8}{|c|}{ Ascospores } & \multicolumn{3}{|c|}{ Signification } \\
\hline & $\begin{array}{l}x 1 \\
(+)\end{array}$ & $\begin{array}{l}x 2 \\
(-)\end{array}$ & $\begin{array}{l}x 3 \\
(-)\end{array}$ & $\begin{array}{c}x 4 \\
(-)\end{array}$ & $\begin{array}{l}x 5 \\
(+)\end{array}$ & $\begin{array}{l}x 6 \\
(-)\end{array}$ & $\begin{array}{l}x 7 \\
(+)\end{array}$ & $\begin{array}{l}x 8 \\
(+)\end{array}$ & Fcal & $\mathrm{F} 0,01$ & ppds à 0,01 \\
\hline Camp Rémy & $4,89^{a}$ & $8,35^{\text {de }}$ & $9,36^{e}$ & $7,39 \mathrm{~cd}$ & $6,64^{b c}$ & $5,78^{a b}$ & $10,67^{f}$ & $6,77^{\mathrm{bc}}$ & 27,92 & 3,02 & 1,25 \\
\hline Étoile de Choisy & $6,56^{a}$ & $9,94^{\mathrm{C}}$ & $11,85^{d}$ & $9,83^{c}$ & $6,14^{a}$ & $8,92^{b c}$ & $9,98^{c}$ & $8,27^{b}$ & 19,33 & 3,02 & 1,54 \\
\hline Lignée $L_{22}$ & $4,06^{a}$ & $5,61^{b}$ & $5,15^{b}$ & $5,27^{b}$ & $3,61^{a}$ & $4,17^{a}$ & $5,04^{b}$ & $4,25^{a}$ & 10,49 & 3,02 & 0,76 \\
\hline
\end{tabular}

Les lettres indiquent les groupes de différence pour la ppds à 0,01 .

Tableau IIIb. Valeur du $t$ de la différence et sa signification entre groupes d'incompatibilité sexuelle et entre ascospores jumelles appartenant à une même paire. Analyse des données utilisées pour établir le tableau Illa.

\begin{tabular}{|c|c|c|c|c|c|c|}
\hline \multirow[t]{2}{*}{ Comparaisons effectuées } & \multicolumn{2}{|c|}{ Sur Camp Rémy } & \multicolumn{2}{|c|}{ Sur Étoile de Choisy } & \multicolumn{2}{|c|}{ Sur $L_{22}$} \\
\hline & $\mathrm{t} \mathrm{cal}$ & Signif & $\mathrm{t} c a l$ & Signif & $\mathrm{t} c \mathrm{cal}$ & Signif \\
\hline $\begin{array}{l}\text { Groupes d'incompatibilité } \\
(6 \mathrm{ddl})\end{array}$ & 0,333 & NS & 2,335 & $>0,05$ & 1,863 & $>0,1$ \\
\hline $\begin{array}{l}\text { Ensemble des paires } \\
=\text { effet mitose }(3 \mathrm{ddl})\end{array}$ & 3,232 & $>0,025$ & 3,382 & $>0,025$ & 2,786 & $>0,05$ \\
\hline \multicolumn{7}{|l|}{ Par paire (14 ddl) } \\
\hline $\begin{array}{l}x 1 \text { et } x 8 \\
(+) \quad(+)\end{array}$ & 4,523 & $>0,001$ & 2,394 & $>0,025$ & 0,867 & $>0,2$ \\
\hline $\begin{array}{l}\times 5 \text { et } \times 7 \\
(+) \quad(+)\end{array}$ & 8,985 & $>0,001$ & 5,063 & $>0,001$ & 4,768 & $>0,001$ \\
\hline $\begin{array}{l}\times 2 \text { et } \times 3 \\
(-) \quad(-)\end{array}$ & 1,503 & $>0,1$ & 3,036 & $>0,005$ & 1,046 & $>0,2$ \\
\hline $\begin{array}{l}x 4 \text { et } x 6 \\
(-) \quad(-)\end{array}$ & 3,208 & $>0,005$ & 2,177 & $>0,025$ & 4,096 & $>0,001$ \\
\hline
\end{tabular}

des valeurs extrêmes est la plus forte sur la variété Camp Rémy. Certaines ascospores apparaissent peu agressives sur les 3 variétés comme $x 1$; par contre, $x 3$ est très agressive. Les ascospores $x 7$ et $x 8$ se classent de façon non constante avec les variétés. Toutefois le coefficient de corrélation de rang entre les classements des ascospores sur Étoile de Choisy et sur Camp Rémy est significatif ( $r s=0,827$ ); il en est de même pour les comparaisons faites avec le classement sur la lignée | 22 ( $r$ s $=0,888$ et $0,91)$.
La comparaison des groupes d'incompatibilité montre une différence au seuil de $5 \%$ sur la variété Étoile de Choisy; les ascospores du groupe (-) donnent des nécroses qui, en moyenne, évoluent plus rapidement que celles provoquées par les ascospores du groupe (+) (tableau IIIb). Cette différence entre les groupes riest décelable qu'au seuil de $10 \%$ sur la lignée $L_{22}$; elle n'existe pas sur la variété Camp Rémy. La comparaison des ascospores jumelles pour toutes les paires prises en compte indique, pour les 3 variétés, des différences qui sont significatives 
au seuil d'au moins $5 \%$. Paire par paire, des différences sont toujours constatées sur Étoile de Choisy, pour 3 paires sur 4 sur Camp Rémy et pour seulement 2 paires sur la lignée $L_{22}$. Sur cet hôte, les ascospores $x 1$ et $x 8$ apparaissent identiques entre elles, de même les ascospores $x 2$ et $x 3$; ces deux paires appartiennent chacune à un groupe d'incompatibilité différent.

Sur les variétés Étoile de Choisy et $\mathrm{CBS}_{1}$, nous avons comparé le classement des ascospores (données du tableau IVa); le coefficient de corrélation de rang n'est pas significatif : $r s=$
0,09 . Sur ces 2 variétés, il existe des différences entre les ascospores, mais on ne peut distinguer les groupes d'incompatibilité entre eux (tableau IVb). Comme dans l'essai précédent, les paires prises ensemble confirment que les ascospores jumelles sont différentes entre elles sur Étoile de Choisy; ce résultat se retrouve pour chacune des 4 paires (tableau IVb). Par contre, sur la lignée CBS1, les paires d'une part $x 5$ et $x 7$ (groupe +), d'autre part $\times 2$ et $\times 3$ (groupe -) associent des ascospores non significativement différentes entre elles. Ce résultat ne se retrouve pas sur les 2 autres paires.

Tableau IVa. Intensité des nécroses foliaires 5 jours après l'inoculation à $17^{\circ} \mathrm{C}$ de fragments foliaires de 2 variétés de blé maintenus en survie (échelle de 0 à 5).

\begin{tabular}{|c|c|c|c|c|c|c|c|c|c|c|c|}
\hline \multirow[t]{2}{*}{ Variétés } & \multicolumn{8}{|c|}{ Ascospores } & \multicolumn{3}{|c|}{ Signification } \\
\hline & $\begin{array}{l}x 1 \\
(+)\end{array}$ & $\begin{array}{l}x 2 \\
(-)\end{array}$ & $\begin{array}{l}x 3 \\
(-)\end{array}$ & $\begin{array}{l}x 4 \\
(-)\end{array}$ & $\begin{array}{l}x 5 \\
(+)\end{array}$ & $\begin{array}{l}x 6 \\
(-)\end{array}$ & $\begin{array}{l}x 7 \\
(+)\end{array}$ & $\begin{array}{l}x 8 \\
(+)\end{array}$ & F cal & $F 0,01$ & $\begin{array}{c}p p d s \\
0,01\end{array}$ \\
\hline $\begin{array}{l}\mathrm{CBS}_{1} \\
\text { (9 répétitions) }\end{array}$ & $2,61^{b}$ & $1,49^{a}$ & $1,57^{a}$ & $3,28^{b}$ & $0,77^{a}$ & $1,03^{a}$ & $0,88^{a}$ & $1,56^{\mathrm{a}}$ & 11,96 & 2,95 & 0,856 \\
\hline $\begin{array}{l}\text { Étoile de Choisy } \\
\text { (5 répétitions) }\end{array}$ & $1,77^{a}$ & $2,66^{b}$ & $3,15^{c}$ & $3,83^{d}$ & $1,75^{\mathrm{a}}$ & $3,15^{c}$ & $3,91^{d}$ & $3,97^{d}$ & 49,05 & 3,25 & 0,331 \\
\hline
\end{tabular}

Les lettres indiquent les groupes de différence pour la ppds à 0,01 .

Tableau IVb. Valeur du $t$ de la différence et sa signification entre groupes d'incompatibilité sexuelle et entres ascospores jumelles appartenant à une même paire. Analyses des données utilisées pour établir le tableau IV.

\begin{tabular}{|c|c|c|c|c|}
\hline \multirow[t]{2}{*}{ Comparaisons effectuées } & \multicolumn{2}{|c|}{ Sur CBS1 } & \multicolumn{2}{|c|}{ Sur Étoile de Choisy } \\
\hline & t cal & Signif & $\mathrm{t} c a l$ & Signif \\
\hline $\begin{array}{l}\text { Groupe d'incompatibilité } \\
\text { (6 ddl) }\end{array}$ & 0,599 & NS & 0,523 & NS \\
\hline $\begin{array}{l}\text { Ensemble des paires } \\
=\text { effet mitose }(3 \mathrm{ddl})\end{array}$ & 1,701 & $>0,1$ & 2,995 & $>0,05$ \\
\hline $\begin{array}{l}\text { Parpaire } \\
\begin{array}{rr}\times 1 & \text { et } \times 8 \\
(+) & (+)\end{array}\end{array}$ & 2,536 & $>0,025$ & 15,44 & $>0,001$ \\
\hline $\begin{array}{l}x 5 \text { et } \times 7 \\
(+) \quad(+)\end{array}$ & 0,513 & NS & 55,51 & $>0,001$ \\
\hline $\begin{array}{l}\times 2 \text { et } \times 3 \\
(-) \quad(-)\end{array}$ & 0,207 & NS & 2,693 & $>0,025$ \\
\hline $\begin{array}{l}x 4 \text { et } \times 6 \\
(-) \quad(-)\end{array}$ & 5,704 & $>0,001$ & 5,179 & $>0,05$ \\
\hline
\end{tabular}




\section{Quantités d'éthylène produit après contamination d'Étoile de Choisy}

Cette production, au cours du temps, est représentée pour les fragments témoins non inoculés et pour 2 paires d'ascospores appartenant chacune à un groupe différent d'incompatibilité sexuelle (fig 1). Pour ce marqueur physiologique des relations hôte-parasite toutes les souches se distinguent du témoin, et c'est à $72 \mathrm{~h}$ après la contamination que les discriminations obtenues entre les ascospores sont les plus grandes (tableau $\mathrm{Va}$ ). Les différences observées se retrouvent au niveau de la note moyenne attribuée pour caractériser les nécroses. Entre ces notes et les quantités d'éthylène produites, il existe une régression linéaire dont le coefficient de corrélation est très élevée. À $72 \mathrm{~h}$, il vaut 0,948 : éthylène et nécrose varient dans le même sens. Pour les ascospores regroupées par groupes d'incompatibilité on en constate jamais de différences significatives entre ces groupes (tableau $\mathrm{Vb})$, de même que pour les nécroses foliaires relevées aux diverses périodes de temps. Pour les paires de jumelles, prises ensemble, des différences sont détectées, pour la quantité d'éthy-

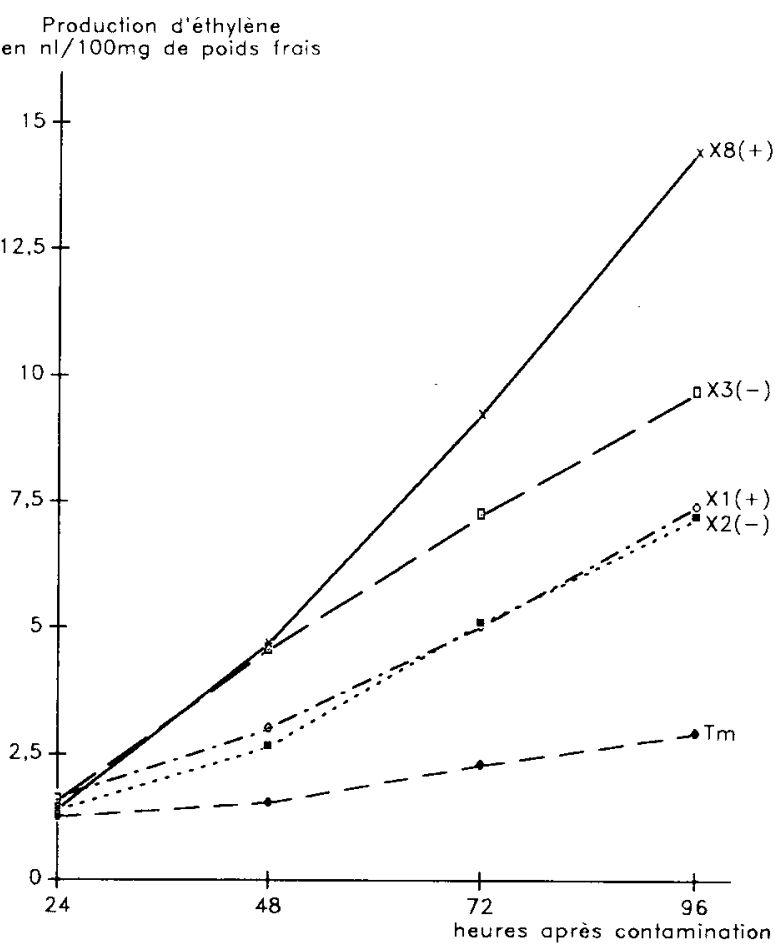

Fig 1. Production d'éthylène exprimée en $\mathrm{nl}$ pour $100 \mathrm{mg}$ de poids frais de fragments de feuille d'Étoile de Choisy en fonction du temps (en h) après la contamination. Comparaisons des ascospores jumelles $x 1$ et $\times 8$ appartenant au groupe d'incompatibilité sexuelle plus $(+)$ et des ascospores jumelles $\mathrm{x} 2$ et $\mathrm{x} 3$ appartenant au groupe d'incompatibilité sexuelle moins $(-)$. Tm $=$ fragments témoins non inoculés (feuilles maintenues en survie à $17{ }^{\circ} \mathrm{C}$, moyenne de 4 répétitions). lène, dès $24 \mathrm{~h}$, après la contamination et en l'absence de nécroses foliaires visibles. Ces différences s'accentuent avec le temps. II en est de même pour les nécroses au-delà de $48 \mathrm{~h}$ après l'inoculation. Paire par paire d'ascospores, les mêmes observations peuvent être formulées. Là aussi, dès $24 \mathrm{~h}$, des différences commencent à apparaître pour l'éthylène et à $48 \mathrm{~h}$ pour les nécroses, sauf pour $\times 2$ et $\times 3$ où la différence n'est significative qu'après $72 \mathrm{~h}$. Pour la paire $x 5$ et $x 7$, les différences dans la production d'éthylène n'apparaissent réellement qu'à partir de $72 \mathrm{~h}$, après la distinction que permettent de réaliser les nécroses foliaires. Enfin, pour $x 4$ et $x 6$, les distinctions faites pour l'éthylène sont toujours de faible niveau de signification.

\section{Comparaisons des ascospores par la quantité de maladie}

Les quantités de maladie induites par les épidémies sont appréciées par l'aire sous la courbe épidémique. Les différences entre les ascospores sont plus faibles que celles relevées pour les nécroses foliaires sur fragments de feuilles en survie. Toutefois, sur la variété Étoile de Choisy, les ascospores peuvent être distinguées entre elles au seuil de 5\% (tableau VI). Les épidémies induites par les ascospores $\times 3$ et $x 7$ sont celles qui provoquent le plus de maladie sur Étoile de Choisy alors que, sur la lignée UPC 5 , c'est celle induite par $x 5$. Pour les 2 variétés, ce critère de l'aire sous la courbe ne permet pas de distinguer les groupes d'incompatibilité. Les paires d'ascospores apparaissent faites d'ascospores identiques entre elles sur Étoile de Choisy; sur la lignée $\mathrm{UPC}_{5}$ il n'est pas possible de considérer les paires comme associant des ascospores jumelles.

\section{Comparaisons de monomacropycnidiospores issues des ascospores $\times 6$ et $\times 8$}

Ces souches sont, pour une même ascospore, issues d'une seule pycnide. Au sein de chacune des deux séries de monospores, des différences sont constatées pour la note d'intensité des nécroses foliaires relevée sur la variété Étoile de Choisy (tableau VII) tant après 6 j qu'après 8 . II apparaît aussi des différences dans le pourcentage de nécroses qui portent des pycnides après $11 \mathrm{j}$. La souche parentale $x 8$ donne des pycnides 
Tableau Va. Production d'éthylène en $\mathrm{nl}$ pour $100 \mathrm{mg}$ de poids frais de fragments foliaires de la variété Étoile de Choisy maintenue en survie à $17^{\circ} \mathrm{C}, 72 \mathrm{~h}$ après l'inoculation par les souches mono-ascospores et intensité des nécroses foliaires (échelle de 0 à $5 ; 4$ répétitions).

\begin{tabular}{|c|c|c|c|c|c|c|c|c|c|c|}
\hline \multirow[t]{2}{*}{ Observations } & \multicolumn{9}{|c|}{ Ascospores } & \multirow[b]{2}{*}{$\begin{array}{r}\text { ppds } \\
0,01\end{array}$} \\
\hline & $T m$ & $\begin{array}{l}x 1 \\
(+)\end{array}$ & $\begin{array}{l}x 2 \\
(-)\end{array}$ & $\begin{array}{l}x 3 \\
(-)\end{array}$ & $\begin{array}{l}x 4 \\
(-)\end{array}$ & $\begin{array}{l}x 5 \\
(+)\end{array}$ & $\begin{array}{l}x 6 \\
(-)\end{array}$ & $\begin{array}{l}x 7 \\
(+)\end{array}$ & $\begin{array}{l}x 8 \\
(+)\end{array}$ & \\
\hline Éthylène & $2,285^{a}$ & $5,01^{b}$ & $5,08^{b}$ & $7,23^{\mathrm{cd}}$ & $7,48^{\mathrm{cd}}$ & $6,95^{c}$ & $8,28^{\text {de }}$ & $8,97^{e}$ & $9,24^{e}$ & 1,39 \\
\hline Nécroses & - & $0,24^{a b}$ & $0,56^{\mathrm{ab}}$ & $2,12^{d}$ & $1,69^{c}$ & $0,87^{\mathrm{b}}$ & $2,31^{d}$ & $2,94^{\mathrm{e}}$ & $3,04^{e}$ & 0,32 \\
\hline
\end{tabular}

Coefficient de corrélation linéaire entre éthylène et nécrose $r=0,948$ THS. Les lettres indiquent les groupes de différence pour la ppds à 0,01 .

Tableau $\mathrm{Vb}$.Valeur du $t$ de la différence et sa signification entre groupes d'incompatibilité sexuelle et entre ascospores jumelles appartenant à une même paire, pour la production d'éthylène et pour l'intensité des nécroses à diverses périodes de temps après l'inoculation.

\begin{tabular}{|c|c|c|c|c|c|c|c|c|}
\hline \multirow{3}{*}{$\begin{array}{l}\text { Comparaisons } \\
\text { effectuées }\end{array}$} & \multicolumn{8}{|c|}{ Heures après la contamination } \\
\hline & \multicolumn{2}{|r|}{24} & \multicolumn{2}{|c|}{48} & \multicolumn{2}{|r|}{72} & \multicolumn{2}{|c|}{96} \\
\hline & t cal & Signif & t cal & Signif & $\mathrm{t} c a l$ & Signif & t cal & Signif \\
\hline \multicolumn{9}{|c|}{$\begin{array}{l}\text { Groupe d'incompatibilité } \\
(6 \mathrm{ddl})\end{array}$} \\
\hline Nécrose & - & - & 0,945 & $>0,2$ & 0,123 & NS & 0,649 & NS \\
\hline Éthylène & 0,16 & NS & 0,545 & NS & 0,439 & NS & 0,502 & NS \\
\hline \multicolumn{9}{|c|}{$\begin{array}{l}\text { Ensemble des paires } \\
=\text { effet mitose }(3 d d)\end{array}$} \\
\hline Nécrose & - & - & 1,702 & $>0,1$ & 2,911 & $>0,05$ & 2,963 & $>0,05$ \\
\hline Éthylène & 8,462 & $>0,005$ & 2,278 & $>0,075$ & 6,245 & $>0,005$ & 2,775 & $>0,05$ \\
\hline \multicolumn{9}{|l|}{ Par paire } \\
\hline \multirow{2}{*}{$\begin{array}{l}\text { x1 et x8 nécrose } \\
(+)(+) \\
\text { éthylène }\end{array}$} & - & & 25,148 & $>0,001$ & 29,413 & $>0,001$ & 10,459 & $>0,001$ \\
\hline & 1,591 & $>0,1$ & 4,496 & $>0,005$ & 6,768 & $>0,001$ & 7,164 & $>0,001$ \\
\hline \multirow{2}{*}{$\begin{array}{l}x 5 \text { et } x 7 \text { nécrose } \\
(+)(+) \\
\text { éthylène }\end{array}$} & - & & 29,43 & $>0,001$ & 17,622 & $>0,001$ & 5,236 & $>0,001$ \\
\hline & 1,504 & $>0,1$ & 1,322 & $>0,15$ & 3,961 & $>0,005$ & 5,744 & $>0,001$ \\
\hline \multirow{2}{*}{$\begin{array}{l}\text { x2 et x3 nécrose } \\
(-) \quad(-) \\
\text { éthylène }\end{array}$} & - & & 0,419 & NS & 15,048 & $>0,001$ & 2,502 & $>0,025$ \\
\hline & 1,91 & $>0,1$ & 14,662 & $>0,001$ & 9,219 & $>0,001$ & 4,21 & $>0,005$ \\
\hline \multirow{2}{*}{$\begin{array}{l}x 4 \text { et } \times 6 \text { nécrose } \\
(-) \quad(-) \\
\text { éthylène }\end{array}$} & - & & 1,667 & $>0,1$ & 3,693 & $>0,005$ & 8,385 & $>0,001$ \\
\hline & 0,976 & $>0,15$ & 0,09 & NS & 1,565 & $>0,1$ & 1,194 & $>0,15$ \\
\hline
\end{tabular}

dans un délai toujours plus court que la souche x6. II y a donc globablement une transmission de l'agressivité à la descendance. Alors qu'au sein de chaque série de monomacropycnidiospores on ne peut relever de différences pour les caractères culturaux, le contenu d'une pycnide issue 
Tableau VI. Quantités de maladie (appréciées par l'aire sous la courbe épidémique) provoquées en 58 jours par les épidémies initiées avec les souches monoascospores (moyenne de 2 répétitions). (a). Valeur du $t$ de la différence et sa signification entre groupes d'incompatibilité sexuelle et entre ascospores jumelles associées par paire (b).

(a)

Variétés

Ascospores

\begin{tabular}{ccccccccc}
\hline $\begin{array}{c}x 1 \\
(+)\end{array}$ & $\begin{array}{c}x 2 \\
(-)\end{array}$ & $\begin{array}{c}x 3 \\
(-)\end{array}$ & $\begin{array}{c}x 4 \\
(-)\end{array}$ & $\begin{array}{c}x 5 \\
(+)\end{array}$ & $\begin{array}{c}x 6 \\
(-)\end{array}$ & $\begin{array}{c}x 7 \\
(+)\end{array}$ & $\begin{array}{c}x 8 \\
(+)\end{array}$ & ppds \\
$972^{\mathrm{a}}$ & $1027^{\mathrm{a}}$ & $1289^{\mathrm{b}}$ & $890^{\mathrm{a}}$ & $1095^{\mathrm{ab}}$ & $1080^{\mathrm{ab}}$ & $1224^{\mathrm{b}}$ & $854^{\mathrm{a}}$ & 290 à 0,05 \\
$805^{\mathrm{ab}}$ & $770^{\mathrm{ab}}$ & $698^{\mathrm{ab}}$ & $669^{\mathrm{ab}}$ & $842^{\mathrm{b}}$ & $632^{\mathrm{a}}$ & $768^{\mathrm{ab}}$ & $655^{\mathrm{ab}}$ & 209 à 0,1
\end{tabular}

(b)

$\frac{\text { Étoile de Choisy }}{\mathrm{t} \text { cal Signification }} \frac{\mathrm{t} \text { cal } \quad \text { Signées } \mathrm{UPC}_{5}}{}$

Comparaisons des groupes

d'incompatibilité sexuelle $(6 \mathrm{ddl})$

$\begin{array}{llll}0,424 & N S & 1,109 & >0,25\end{array}$

Comparaison de l'ensemble des

paires d'ascospores jumelles

1,109

$>0,25$

$=$ effet mitose $(3 \mathrm{ddl})$

$\begin{array}{llll}1,502 & >0,15 & 3,544 & >0,025\end{array}$

Tableau VIl. Comparaisons par l'intensité des nécroses foliaires (échelle de 0 à 5) relevée 6 à 8 j (à $17^{\circ} \mathrm{C}$ ) après l'inoculation par des monomacropycnidiospores issues d'une seule pycnide différenciée à partir de la monoascospore $x 6$ ou $x 8$. Pourcentage de nécroses portant des pycnides après 11 j (30 répétitions sur fragments de feuille d'Étoile de Choisy).

Origne des

monomacropycnidiospores
Intensité des nécroses

$6 j \quad 8 j$

Pourcentage de nécroses portant des pycnides après $11 j$

\section{Ascospore $\times 6$ pycnide $P_{3}$}

$$
\begin{aligned}
& x 6-P_{3}-4 \\
& x 6-P_{3}-5 \\
& x 6-P_{3}-8 \\
& x 6-P_{3}-19 \\
& x 6-P_{3}-21
\end{aligned}
$$

ppds 0,05

Ascospore $x 8$, pycnide $P_{1}$

$$
\begin{aligned}
& x 8-P_{1}-1 \\
& x 8-P_{1}-2 \\
& x 8-P_{1}-3 \\
& x 8-P_{1}-4 \\
& x 8-P_{1}-6
\end{aligned}
$$

ppds 0,05

$1,95^{\mathrm{c}}$
$0,89^{\mathrm{ab}}$
$0,9^{\mathrm{b}}$
$0,81^{\mathrm{a}}$
$1,11^{\mathrm{b}}$

0,45

0,34

0,51 
Tableau VIII. Nombre de fois où les paires théoriques d'ascospores associent dans les essais 2 ascospores non différentes entre elles au seuil de 5\% (pour l'éthylène, seules les observations faites à 72 h sont prises en compte).

Paires théoriques du groupe +

\begin{tabular}{|c|c|c|c|c|c|}
\hline Paire & Identique & Différent & Paire & Identique & Différent \\
\hline$x 1-x 5$ & 2 & 5 & $x 2-x 3^{*}$ & 3 & 4 \\
\hline$x 1-x 7$ & 0 & 7 & $x 2-x 4$ & 1 & 6 \\
\hline$x 1-x 8^{*}$ & 1 & 6 & $x 2-x 6$ & 1 & 6 \\
\hline$x 5-x 7^{\star}$ & 1 & 6 & $x 3-x 4$ & 2 & 5 \\
\hline$x 5-x 8$ & 2 & 5 & $x 3-x 6$ & 2 & 5 \\
\hline$x 7-x 8$ & 2 & 5 & $x 4-x 6^{*}$ & 1 & 6 \\
\hline
\end{tabular}

* Paires associants des ascospores identiques pour leurs caractères culturaux et la pigmentation mycélienne.

d'une ascospore apparaît hétérogène pour le critère d'agressivité pris en compte.

\section{DISCUSSION ET CONCLUSIONS}

La reproduction sexuée de Phaeosphaeria nodorum permet d'obtenir des ascospores dont les niveaux d'agressivité sont très différents entre eux. Regroupées par allèle d'incompatibilité sexuelle, les ascospores apparaissent identiques entre elles, sauf dans un cas, sur Étoile de Choisy. Pour les critères utilisés sur feuilles en survie (intensité des nécroses, production d'éthylène) et pour la quantité de maladie produite en champ, il n'est pas possible d'associer une agressivité particulière à tel ou tel groupe d'incompatibilité sexuelle, alors qu'au sein de chaque groupe l'amplitude de l'agressivité est du même ordre de grandeur. Pour une virulence monogénique chez Venturia inaequalis, Keitt et Langford (1941) arrivaient à la même conclusion.

La troisième division nucléaire, au sein de l'asque, est une mitose haploïde qui permet la formation de huit noyaux correspondant aux quatre paires d'ascospores regroupant des jumelles nucléaires identifiables par l'aspect général, in vitro, des colonies (disposition et couleur des pycnides, des cirrhes, diffusion de pigments dans la gélose) et surtout, par la pigmentation mycélienne. Ces caractères sont les seuls dont on dispose pour identifier les jumelles. Chez Venturia inaequalis, Keitt et Langford (1941) ont utilisé ce type de caractère pour identifier les paires et, dans ce cas, il y a concordance avec le pouvoir pathogène étudié. Dans ce cas de $P$ nodo- rum, force est de constater, sur la base des critères relevés pour juger de l'agressivité, qu'il n'est pas possible de reconnaître les paires d'ascospores jumelles. II n'y a donc aucun lien entre caractères culturaux et agressivité de $P$ nodorum. Au sein de chaque groupe d'incompatibilité, il existe 6 façons théoriques d'associer les ascospores par deux mais, comme le montre le tableau VIII, aucune de ces associations n'apparaît supérieure à celles réalisées sur la base des caractères culturaux. Notons qu'il en est de même pour la réduction du poids de 1000 grains (données non publiées). Cette impossibilité de reconnaître le produit de la dernière mitose pour l'agressivité pose de nombreuses interrogations. Les différences entre les ascospores jumelles doivent exister très tôt puisque $24 \mathrm{~h}$ après l'inoculation, elles sont décelables par la production d'éthylène qui caractérise l'intensité des relations physiologiques entre l'hôte et le parasite, alors qu'il n'y a pas de nécroses foliaires visibles.

Entre cette dernière mitose et les suspensions conidiennes utilisées pour réaliser les inoculations, de très nombreuses mitoses haploïdes ont eu lieu. Les premières se déroulent dans l'asque lui-même pour la différenciation finale des ascospores qui sont formées de 4 cellules uninucléées (Halama, 1991). D'autres ont lieu lors de la germination des ascospores qui, le plus souvent, germent par les cellules des extrémités. Les souches utilisées sont donc issues d'au moins 2 filaments germinatifs. Si des anomalies mitotiques, des délétions, des mutations... ont lieu au cours de ces mitoses successives, il peut $y$ avoir, après anastomose entre 
filaments mycéliens, formation de l'équivalent d'hétérocaryons qui peuvent être à l'origine d'une certaine variabilité, même si l'hétérocaryose est toujours rompue lors de la formation des pycnidiospores (Newton et Caten, 1985).

Si de telles anomalies nucléaires ont lieu lors de ces mitoses successives, elles peuvent se révéler lors de nos essais et, dans ce cas, la descendance d'une ascospore doit être hétérogène. C'est ce que montrent les 2 séries de monospores $\times 6 P_{3}$ et $\times 8 P_{1}$ qui appartiennent à l'un ou l'autre des groupes d'incompatibilité sexuelle. Rappelons que Skajennikoff et Rapilly (1989) avaient montré la grande variabilité, pour l'agressivité, de la descendance monomacropycnidiospore d'une souche sauvage; elle est du même ordre de grandeur que celles observées pour les descendances asexuées des souches monoascospores. L'hétérogénéité du contenu d'une pycnide issue d'une ascospore, que révèle le tableau VII, implique qu'un inoculum issu d'une même ascospore ne doit pas rester identique à lui-même au cours des repiquages successifs. Dans ce cas, le rang de classement des ascospores pour une même variété doit changer d'un essai à l'autre. C'est ce que l'on constate pour les essais réalisés, dans les mêmes conditions expérimentales, sur Étoile de Choisy; le coefficient de corrélation de rang entre le classement des ascospores sur cette variété pour les données des tableaux Illa et Va n'est pas significatif $(r=0,311)$. On doit alors s'interroger sur la signification biologique des interactions constatées entre hôtes et souches monoascosporées, qui sont significatives pour le tableau Illa ( $F$ cal = 4,12; $F 0,05=1,7)$ comme pour les données du tableau IVa ( $F \mathrm{cal}=3,38 ; F 0,05=2,08)$. On doit aussi s'interroger sur la signification de la variabilité de l'expression de l'agressivité que tous les auteurs travaillant sur ce parasite attribuent aux conditions climatiques alors que dans nos essais conduits in vitro, les conditions expérimentales ne changent pratiquement pas. Toutefois, les résultats présentés ci-dessous ne concernent que l'analyse du contenu d'une seule ascospore. Cette variabilité de la descendance ne s'observe pas au niveau des allèles d'incompatibilité sexuelle. Ceux-ci se révèlent stables au cours des générations successives réalisées in vitro ou in vivo et même après pressions de sélections exercées par des changements d'hôtes (Rapilly et al, 1989).

L'instabilité de la descendance monospore a aussi été observée chez Septoria avenae, parasite très proche de $S$ nodorum; Hooker (1957) montre que même après 6 à 8 générations de monospores successives, une gamme d'agressivité peut être obtenue à partir d'une seule monomacropycnidiospore.

Le croisement étudié ici a été réalisé à partir de souches parentales qui semblent très éloignées l'une de l'autre. Cet éloignement peut expliquer aussi l'instabilité de la descendance et le fait de ne pas reconnaître, pour l'agressivité, les ascospores jumelles nucléaires. Toutefois, des observations allant dans le même sens ont été faites par Halama (1991) sur d'autres croisements.

Cooley et Caten (1991), en étudiant le caryotype de divers isolats de $S$ nodorum, constatent d'une part des différences entre isolats provenant de l'hôte blé, d'autre part un nombre très élevé de chromosomes, de 15 à 19, par rapport à d'autres champignons phytopathogènes. Ils détectent aussi la présence de «mini» chromosomes et s'interrogent sur leur rôle dans la variabilité du parasite.

Une autre hypothèse peut être formulée : I'hérédité de l'agressivité ne serait pas seulement nucléaire. Elle pourrait être d'origine nucléocytoplasmique ou cytoplasmique. Jinks (1966) donne de nombreux exemples de champignons où l'hérédité de caractères autres que ceux du pouvoir pathogène n'est pas nucléaire. Kistler et Leong (1986) associent la spécificité d'hôtes de certaines races de Fusarium oxysporum $f \mathrm{sp}$ conglutinans à la présence de plasmides. Garber et al (1984) observent des plasmides chez Cochliobolus heterostrophus mais ils n'ont pu démontrer leur transmission par la reproduction sexuée. Pour expliquer l'adaptation d'Erysiphe graminis $\mathrm{f} \mathrm{sp}$ hordei à des orges à résistance partielle élevée, Newton et Mac Gurk (1991) avancent l'hypothèse de la variabilité cytoplasmique entre oïdies.

Le brassage de cytoplasme est possible chez Septoria nodorum lors de la réalisation d'anastomoses entre mycélium et peut concourir à l'hétérogénéité constatée. Cette dernière hypothèse peut expliquer pourquoi un seul passage sur un nouvel hôte est suffisant pour que l'effet de la pression de sélection exercée par le noyau ou le cytoplasme de l'hôte puisse être mis en évidence (Rapilly et al, 1989).

L'hérédité de l'agressivité, par la reproduction sexuée, de $P$ nodorum apparaît très complexe. Les causes permettant d'expliquer, d'une part, la non-identification pour le pouvoir pathogène des ascospores jumelles et, d'autre part, l'hétérogé- 
néité de la descendance d'une ascospore sont à rechercher. Elles conditionnent l'efficacité du travail des sélectionneurs pour la résistance à la septoriose.

Une voie de recherche à explorer est l'étude de la descendance de croisements dans les deux sens en réalisant la spermatisation par du mycélium, des macropycnidiospores et des micropycnidiospores. Halama (1991) a montré que chacun de ces 3 "éléments" qui représentent des quantités de cytoplasme décroissantes peuvent être utilisés pour féconder une souche de signe sexuel contraire. Mais les contraintes techniques pour l'analyse des ascospores ne permettent d'étudier, dans le même temps, que le contenu de très peu d'asques.

\section{RÉFÉRENCES}

Cooley N, Caten CE (1991) Variation in electrophoretic karyotype between strains of Septoria nodorum. Mol Gen Genet 228, 17-23

Emerson S (1966) Mechanisms in inheritance. In: The fungi : fungal organism (Ainsworth, Sussman, eds) Acad Press, New York, II, 513-566

Garber RC, Turgeon BC, Yoder OC (1984) A mitochondrial plasmid from the plant pathogenic fungus Cochliobolus heterostrophus. Mol Gen Genet 196, 301-310

Halama P (1991) Phaeosphaeria nodorum Hedj (Ex Leptosphaeria nodorum Mull) télémorphe de Septoria nodorum Berk. Thèse Dr Univ Sc Tech de Lille Artois

Halama $P$, Lacoste $L$ (1990) Déterminisme de la reproduction sexuée de Phaeosphaeria nodorum (ex Leptosphaeria nodorum), agent de la septoriose du blé. I. Hétérothallisme et rôle des microspores. Can J Bot 69, 55-99

Hooker AL (1957) Cultural variability in Septoria avenae through successive single macrospore transfer. Phytopathology 47, 460-468

Jinks TL (1966) Mechanisms of inheritance: extra nuclear inheritance. In: The fungi: fungal organism (Ainsworth, Sussman, eds) Acad Press, New York, 619-660
Keitt GW, Langford MA (1941) Venturia inaequalis: a groundwork for genetic studies. Am J Bot 28, 805820

Kistler HC, Leng SA (1986) Linear plasmid-like DNA in the plant pathogenic fungus Fusarium oxysporum $f \mathrm{sp}$ conglutinans. J Bacteriol 167, 587-593

Krupinsky JM (1982) Comparative pathogenicity of Septoria nodorum isolated from Triticum aestivum and Agropyrum species. Phytopathology 72, 660661

Nelson RL, Marshall D (1990) Breeding wheat for resistance to Septoria nodorum and Septoria tritici. Adv Agron 44, 257-277

Newton AC, Caten CE (1985) Heterokaryosis and heterokaryon incompatibility in $S$ nodorum. Septoria of cereals US Dept Agric ARS 12, 135-145

Newton AC, Mc Gurk L (1991) Recurrent selection for adaptation of Erysiphe graminis $\mathrm{f} s p$ hordei to partial resistance and the effect of environment in expression of partial resistance of barley. J Phytopathol 132, 328-338

Osbourn AE, Scott PR, Caten CE (1986) The effects of host passaging on the adaptation of Septoria nodorum to wheat or barley. Plant Pathol 35, 135145

Rapilly F, Foucault B, Lacazedieux J (1973) Études sur l'inoculum de Septoria nodorum Berk (Leptosphaeria nodorum Mull), agent de la septoriose du blé. I. Les ascospores. Ann Phytopathol 5, 131141

Rapilly F, Richard H, Skajennikoff M, Cauderon $Y$, Roussel J (1989) Pressions de sélection exercées par le noyau ou le cytoplasme de l'hôte sur l'agressivité d'un parasite nécrotrophe du blé (Septoria nodorum Berk). Agronomie 9, 703-718

Skajennikoff M, Rapilly F (1989) Variabilité du pouvoir pathogène chez Septoria nodorum Berk. Agronomie 9, 673-702

Wendland M, Hoffmann GM (1987) Proff of quantitative resistance of wheat genotypes to Septoria nodorum by determining post infectional ethylene production. J Plant Dis Prot 94, 561-571

Wendland M, Hoffmann GM (1988) Differentiation of the quantitative resistance of wheat cultivar and lines to Septoria nodorum on the principle of postinfectional ethylene production. J Plant Dis Prot 95, 113-123 